\title{
El desarrollo de la sanidad forestal en el vivero de TRAGSA
}

\author{
Cuenca Valera, B. ${ }^{1 *}$, Lario Leza, F.J. ${ }^{1}$, Luquero Ramos, L. ${ }^{2}$, Ocaña Bueno, L. ${ }^{2}$ \\ ${ }^{1}$ Vivero de TRAGSA. Crta. Maceda-Baldrei, km. 2. 32700 Maceda, Ourense. \\ ${ }^{2}$ Departamento de Tecnología de la Información. Subdirección de Soporte Técnico e Innovación. \\ C/ Conde de Peñalver, 84. 28006 Madrid. \\ *Autor para correspondencia: bcuenca@tragsa.es
}

\section{Resumen}

Se presentan los trabajos que realiza el vivero de TRAGSA en el ámbito de la sanidad forestal, en cuanto al desarrollo de Materiales Forestales de Reproducción con tolerancia a enfermedades, en la producción de parasitoides para el control biológico de plagas forestales, y en el desarrollo de metodologías de teledetección para detección temprana, caracterización y seguimiento del estado fitosanitario de las masas forestales. Se describen los trabajos realizados y en curso al respecto de la obtención de MFR de castaño, de encina y alcornoque por tolerancia a Phytophthora cinnamomi, y de Alnus glutinosa por tolerancia a P. alni. Así mismo se presentan los trabajos de estudio de Torymus sinensis para la lucha biológica contra Dryocosmus kuriphilus, y de producción de Anaphes nitens para la lucha biológica contra Gonipterus platensis. Por último se comentan los trabajos realzados con RPAS para detección y seguimiento de Armillaria mellea en masa de Pinus pinaster.

Palabras clave: tolerancia, resistencia, tinta, seca, Phytophthora, castaño, alcornoque, encina, Quercus, Dryocosmus, Torymus, Anaphes, Gonipterus, teledetección, RPAS, GNDVI, NDVI. 


\section{Introducción}

El grupo TRAGSA es un conjunto de empresas públicas, y por tanto instrumento propio de la Administración, que trabajan en el ámbito del medio ambiente, infraestructuras, edificación, agua y regadío, sanidad, producción agropecuaria, consultoría y emergencias. El grupo TRAGSA es propietario de un vivero forestal en la localidad de Maceda, en Ourense, donde no sólo se produce planta forestal sino que también se desarrolla la producción de nuevos MFR y se trabaja en innovación forestal. Desde este vivero se trabaja en sanidad forestal en tres ámbitos:

1. El desarrollo de Materiales Forestales de Reproducción (MFR) con tolerancia a enfermedades.

2. La producción de parasitoides para el control biológico de plagas forestales.

3. El desarrollo de métodos de teledetección para el seguimiento del estado sanitario de las masas forestales.

En cuanto al primer punto, desde el año 2002 hasta el 2013 se desarrollaron MFR de castaño con tolerancia a la enfermedad de la tinta (Phytophthora cinnamo$m i)$, y actualmente el vivero está implicado en el desarrollo de MFR de encina y alcornoque con tolerancia a la seca, enfermedad también provocada por $P$. cinnamo$m i$, y de MFR de Alnus glutinosa con tolerancia a P. alni. Respecto a la producción de parasitoides, en el vivero se trabaja en el estudio de Torymus sinensis para el control de la avispilla del castaño y de Anaphes nitens para el control del gorgojo del eucalipto. Por último, en teledetección se han desarrollado metodologías, empleando imágenes de satélite y de RPAS, para el seguimiento de Armillaria mellea en masas de Pinus pinaster.

El objetivo de este trabajo, es presentar someramente los trabajos realizados y en curso en el vivero de TRAGSA, en cada uno de estos ámbitos y los resultados conseguidos hasta ahora.

\section{Desarrollo de MFR de castaño con tolerancia a Phytophthora cinnamomi}

En 2002 se comenzó con la selección de materiales de castaño en campo. La idea de partida era disponer de materiales autóctonos gallegos con tolerancia a la tinta pero que se adaptaran mejor que los híbridos a las condiciones de Galicia interior, puesto que estos son sensibles a heladas tempranas y sequía estival, frecuentes en esta zona. Para ello se hizo una prospección en casi 19000 ha, correspondientes a 513 teselas del Mapa Forestal Nacional, en las que había presencia de castaño como primera o segunda especie. Se puso el foco en que estos castaños estuvieran en las zonas 1 o 2 de distribución de la enfermedad de la tinta según Bouhier (1979). En esas zonas se seleccionaron 206 árboles adultos de más de $50 \mathrm{~cm}$ de diámetro, para garantizar que hubieran sufrido y sobrevivido la afección de la enfermedad. Estos 
genotipos se establecieron in vitro en nuestro laboratorio consiguiendo establecer con éxito 136 genotipos que se cultivaron regularmente para obtener copias suficientes con las que hacer la evaluación de resistencia, el establecimiento de ensayos y la crioconservación.

Para evaluar su resistencia a tinta, se realizaron 3 tipos de test diferentes: el primero por ser el más inmediato, fue el test de estaca escindida con el material directamente recogido del árbol donante (Salesses et al., 1993); al mismo tiempo se desarrolló con la ayuda de la Estación de Fitopatología do Areeiro, un test para llevar a cabo con el material in vitro (Rial et al.,2007), y a medida que se fue disponiendo de planta aclimatada de cada genotipo en cantidad suficiente, se inoculó el sustrato de planta viva según la metodología descrita por Robin et al. (2006) que ya había sido aplicada por los investigadores del CIF Lourizán para evaluar la resistencia de los clones híbridos. Este último test, fue el que se consideró más fiable por respetar el sistema habitual de infección del patógeno. Se registraron datos de supervivencia, de nivel de daño en la parte aérea de la planta (Figura 1), de nivel de necrosis en la raíz, frecuencia de podredumbre de raíz y del cuello de la planta, porcentaje de circunferencia de cuello afectado y la longitud de la lesión en el tallo. Finalmente, se confirmó la presencia del oomiceto reaislándolo de la raíz y del suelo. Al comparar los tres test, se encontraron correlación significativas y altas, entre las variables de velocidad de avance de la lesión (test de estaca) y el tiempo hasta la muerte de la planta (test in vitro y de inoculación del sustrato).

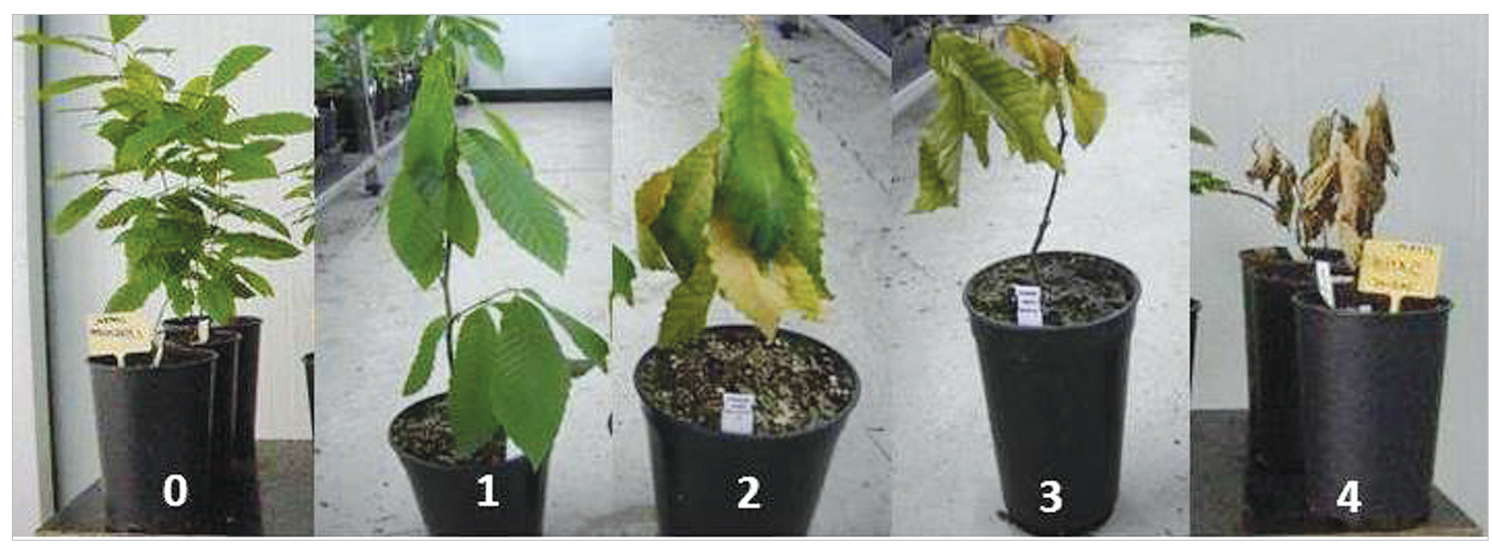

Figura 1. Niveles de daño por Phytophthora cinnamomi en la parte aérea de vitroplantas de castaño: 0, ningún daño hasta 4 , planta muerta.

Entre 2007 y 2010, se evaluaron 92 genotipos empleando Castanea sativa y $C$. crenata clonales como testigos sensibles y resistentes respectivamente. En 2011 se evaluaron juntos los genotipos que mejores resultados obtuvieron en los test realizados a lo largo de los tres años, y se estableció un ranking considerando las dos variables que resultaron ser más discriminantes: nivel de necrosis de la raíz, y el porcentaje de circunferencia del cuello de la raíz afectado de pudrimiento. Respecto al nivel de necrosis de la raíz, se establecían 5 niveles, y respecto al porcentaje de cir- 
cunferencia de cuello con podredumbre, el criterio fue que el valor obtenido no fuera significativamente diferente del producido en C. crenata. De este modo, 7 clones presentaban una circunferencia de pudrición en el cuello no significativamente diferente de C. crenata, y 7 un nivel de necrosis inferior a 2, 6 de los cuales coincidían en ambos criterios. El clon C042 a pesar de diferir significativamente de C. crenata en la circunferencia del cuello, también se seleccionó por su proximidad a $C$. sativa en la caracterización molecular, donde todos los clones salvo C042 y C053, mostraron presencia de alelos asiáticos (Cuenca et al., 2013).

\section{Desarrollo de MFR de encina y alcornoque con tolerancia a la enfermedad de la seca}

En 2014 por interés de la Junta de Extremadura, se presentó un expediente a la convocatoria de Proyectos de Investigación Fundamental Orientada a los Recursos y Tecnologías Agrarias del INIA junto con el CICYTEX, Universidad de Córdoba y el IFAPA, para buscar soluciones al problema de la seca de la encina y del alcornoque. Entre las tareas del vivero de TRAGSA, está la selección de árboles putativamente resistentes y su clonación. Para ello, a partir de un informe previo sobre focos de incidencia de seca de TRAGSATEC, cruzándolo con las regiones y subregiones de procedencia, se preparó una propuesta de 33 puntos de muestreo. Estos puntos se consensuaron con los técnicos del CICYTEX, y en otoño de 2015 fueron visitados por técnicos del ICMC quienes seleccionaron en cada uno de esos puntos, 3 árboles asintomáticos y elaboraron una ficha con la información de cada punto y de los 3 árboles. Se seleccionaron finalmente 84 encinas y 15 alcornoques, y entre Noviembre y Diciembre de 2015, un técnico de TRAGSA visitó cada árbol para la recogida de bellota. En algunos casos, por premura de tiempo fueron los propios técnicos de CICYTEX quienes recogieron la bellota. Finalmente se consiguió bellota de 81 árboles, que fue desinfectada y estratificada en el vivero de TRAGSA hasta su germinación.

En Enero de 2016, se seleccionaron 75 progenitores (67 encinas y 8 alcornoques) de los 81 inicialmente seleccionados en campo, de los que se disponía de cantidad de fruto suficiente para poder realizar el ensayo de evaluación de resistencia. Estas bellotas se sembraron con un diseño de bloques completos al azar con 50 bloques, y cada progenie representada por 2 individuos en cada bloque. Se sembraron también dos bloques control que no se inocularon. En el 10\% de los bloques se controló el peso de la semilla y la fecha de emergencia. En primavera se preparó inóculo según el protocolo de Jung et al. (1996) a partir de un aislado virulento cedido por la U. de Extremadura, previamente reactivado mediante paso por planta viva, y se inoculó según la metodología de Solla et al. (2011), aportando un poco de inoculo en cada alveolo. Las bandejas una vez inoculadas se inundaron hasta el cuello de la raíz durante $24 \mathrm{~h}$ y posteriormente se mantuvieron continuamente en condiciones de saturación. Antes de inocular se controló la altura de los mismos 5 bloques de los que se hizo seguimiento de peso de bellota y fecha 


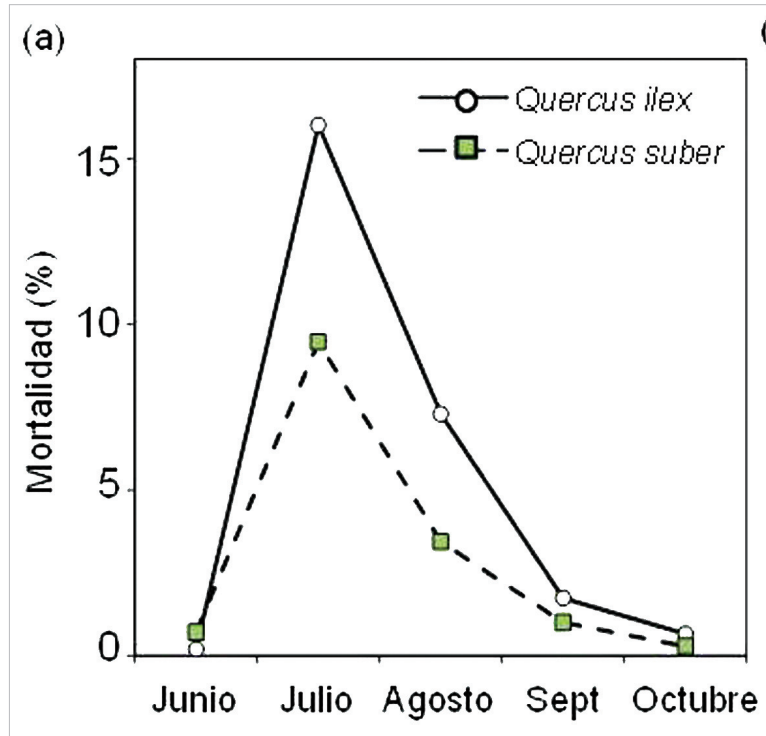

(b)

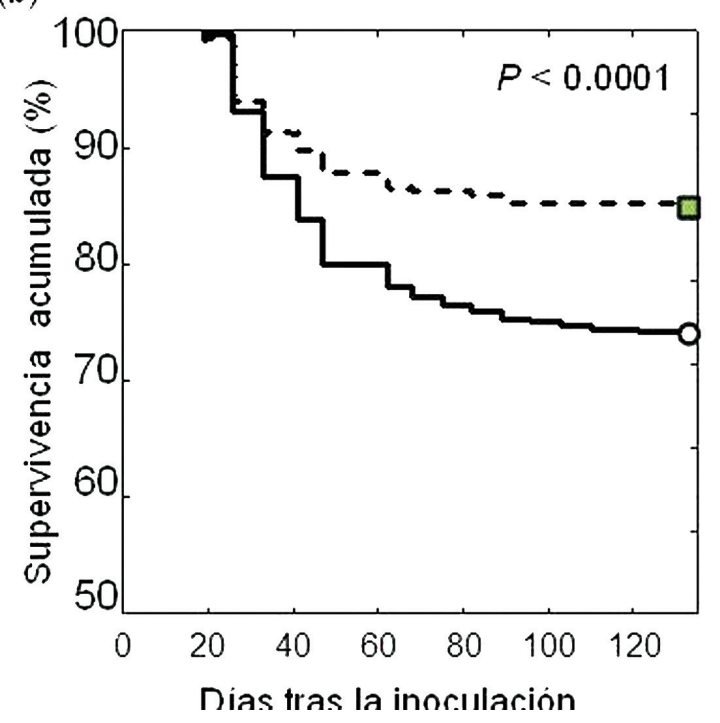

Figura 2. Mortalidad (a) y supervivencia acumulada (b) de Quercus ilex y $Q$. suber tras la inoculación con Phytophthora cinnamomi.

de germinación. La toma de datos de mortalidad se hizo semanalmente desde la inoculación en Junio hasta Octubre de 2016, momento en que las muertes se detuvieron.

A finales de Octubre se observaron mortalidades acumuladas del 25,9\% y 14,9\% en planta de Quercus ilex y Q. suber (Figura 2), con picos de mortalidad en Julio. Los modelos de supervivencia difirieron significativamente entre especies, con esperanzas medias de vida de 109 días para la encina y 118 para el alcornoque. En encina no se apreciaron diferencias significativas entre los materiales de las 5 subregiones de procedencia, ni entre las progenies de Cáceres y Badajoz, pero sí entre las diferentes progenies: la progenie 499 fue la menos susceptible y la 433 la más susceptible. En alcornoque sí se apreciaron diferencias significativas de supervivencia media, tanto entre las regiones de procedencia evaluadas, como entre progenies, con Sierra Morena Occidental con el porcentaje más alto de supervivencia, y la progenie 450 con mortalidad 0 , y la 439 como la más susceptible. Con los datos de mortalidad en Octubre, se elaboró un ranking identificándose cuatro árboles de encina $(499,545,425$, y 504) y cuatro de alcornoque $(450,452,451$ y 438$)$ que mostraron mortalidades inferiores al 5\% tras la inoculación con P. cinnamomi y podrían por tanto, considerarse tolerantes. Lógicamente, estos árboles y sus progenies podrían emplearse para una estrategia de conservación en la dehesa extremeña.

Este trabajo aún está en curso, y en la anualidad 2007, las plantas supervivientes se han reinoculado y sometido a períodos de estrés hídrico, mientras se avanza en la tarea de la micropropagación de ambas especies. 


\section{Desarrollo de MFR de Alnus glutinosa con tolerancia a Phytophthora cinnamomi}

Además de los trabajos con fagáceas y P. cinnamomi, a instancias de la Confederación Hidrográfica del Miño-Sil en el vivero, se está trabajando en la selección de materiales de Alnus glutinosa con tolerancia a $P$. alni. Para ello se han seleccionado 6 cursos de ríos, que presentaban zonas con positivos a $P$. alni, y cada uno de los cursos se han seleccionado 5 árboles asintomáticos de los que se ha recogido material para su establecimiento in vitro y para la realización de un screening previo. Para este screening se emplearon ramillos del año, cuyas bases se pusieron en contacto con zoosporas del aislado UEx23 de P. xalni suministrado por la U. de Extremadura (Chandelier et al., 2015). Los genotipos que presentaron menor marchitamiento en el test fueron seleccionados para su clonación, 2 por curso de agua. Sin embargo, los genotipos de los cursos de Avia y Arnoia mostraron un marchitamiento simultáneo de todas las hojas en unas pocas horas, por lo que con estos genotipos se realizó un segundo test sumergiendo las bases de los ramillos en una suspensión de zoosporas del mismo aislado en agua de rio, y seleccionando aquellos genotipos que presentaran menor longitud de lesión necrótica (A. Solla, pers.comun.). Los genotipos con mejores resultados en estos screenings fueron aquellos en los que se centraron los esfuerzos de clonación. En el caso de Louro, que se seleccionó muy tarde y con el que no se pudo llevar a cabo el screenings, se están clonando los genotipos que han presentado una brotación más vigorosa.

Para evaluar convenientemente la tolerancia de Alnus glutinosa es fundamental disponer de un test con suficiente capacidad de discriminación. Por ello, durante el verano de 2016 se inició la puesta a punto de un test de evaluación de resistencia. Inicialmente se realizó un test de inoculación por inundación del sustrato en el mes de Julio, con el mismo aislado usado en el screenings y siguiendo la misma metodología que en su día empleamos con el castaño (Cuenca et al., 2008). Sin embargo, en el mes de Agosto, la planta no solo no presentaba ningún tipo de síntoma sino que aparecía vigorosa y había crecido ostensiblemente. Por ello, en Agosto se realizó un test de herida en el tallo (Chandelier et al., 2015), pero al cabo de un mes, estas heridas estaban cicatrizando sin presentar lesiones necróticas. En Diciembre de 2016 repetimos este test con las plantas del ensayo que estaba en cámara controlada y por tanto aún vegetaban, empleando un aislado más virulento (Pa4017 de la Universidad de Valladolid). Al cabo de 15 d, los testigos sensibles (planta de semi1la) presentaban pequeñas lesiones necróticas sin diferencias significativas con la planta teóricamente tolerante. El test se repitió en Junio de 2017 con planta en invernadero, obteniendo también ligeras lesiones necróticas únicamente en las plantas de semilla, y sin diferencias significativas, lo que nos ha hecho concluir que este test no arroja resultados discriminantes. El test de inoculación por inundación se repitió también en Junio empleando el aislado Pa4017 pero usando una metodología ya probada en Alnus glutinosa con ligeras modificaciones (Chandelier et al., 2015) con planta tanto en invernadero como en cámara controlada, y alternando períodos de sequía e inundación. 
Ante el aparente fracaso de estos primeros intentos de puesta a punto de un test, se barajaron varias hipótesis: si se dio falta de virulencia del primer aislado empleado; si la enfermedad se desarrolla lentamente y por eso la aparición de síntomas no se produce en el plazo habitual en otras especies con $P$. cinnamomi; o si además de la presencia del patógeno, hay otros factores que determinan que la enfermedad se desencadene y que no se estaban teniendo en cuenta.

En la búsqueda de otros posibles test que produjeran una mayor discriminación, en Junio de 2017 se llevaron a cabo otros dos descritos por Haque et al. (2015). Se realizó un test de inoculación de hojas, donde se midieron las lesiones producidas sobre el limbo de la hoja por un disco de micelio de P. xalni creciendo sobre V8 al cabo de los 11 días. Se obtuvieron lesiones significativamente mayores en los testigos sensibles que en las plantas teóricamente resistentes (Figura 3A). Sin embargo, ocurrió que los controles inoculados en blanco (con un disco de V8 pero sin micelio), tanto de las plantas de semilla como de las clonales tolerantes, presentaron lesiones mucho mayores que las de las hojas inoculadas, lo que tal vez pudo deberse a la presencia de algún saprófito o de Phytium que al no tener que competir con $P$. xalni en los controles, tuvo un mayor desarrollo.

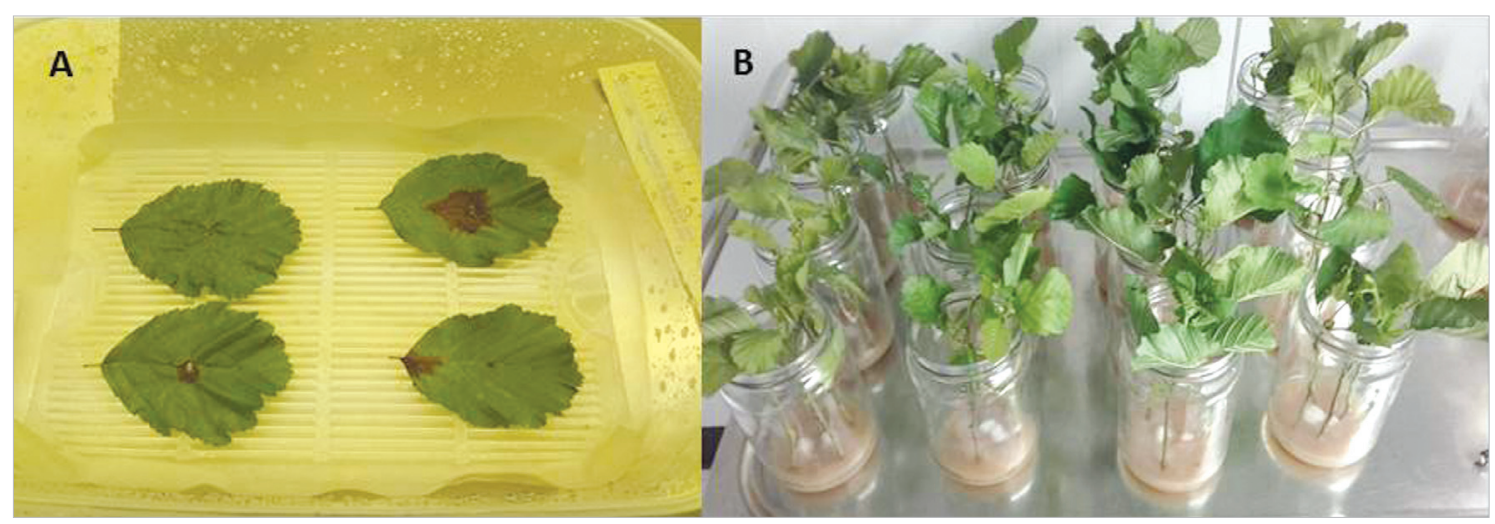

Figura 3. Evaluación de la susceptibilidad de genotipos de Alnus glutinosa a Phytophthora xalni, mediante el test de hoja escindida (A) y el test de ramillos (B)

También se realizó el test de inoculación de ramillos, pero en esta ocasión clavando las bases peladas de los ramillos de $15 \mathrm{~cm}$ en agar V8 con micelio del aislado en crecimiento activo (Figura 3B). Se midieron las longitudes de las lesiones en los ramillos de los genotipos ensayados y en los controles de plantas de semilla, y aunque no se obtuvieron diferencias significativas, la tendencia en el ranking de tolerancia de los 3 genotipos y el control fue similar al del test anterior. Por lo tanto y a la espera de los resultados que pueda arrojar el test de inoculación por inundación, el test que ha resultado más discriminante es el de inoculación de hojas. Cuando se disponga de los resultados del test de inundación, se estudiarán las correlaciones entre las variables de ambos test, para determinar la utilidad del test de hojas como screening preliminar. 


\section{Estudio de Torymus sinensis para el control de la avispilla del castaño}

El primer objetivo en este trabajo, encomienda de la Xunta de Galicia, fue determinar la fenología del triple ciclo implicado en el proceso en las condiciones climáticas del vivero de TRAGSA. Los brinzales y ramets de una savia de castaño, son comercializados tras la caída de la hoja en invierno, sin mostrar ningún tipo de síntoma de la presencia de avispilla. Sin embargo, puede haber ocurrido, que un adulto partenogénico de Dryocosmus kuriphilus haya depositado la primavera anterior uno o varios huevos en una yema, y que en la siguiente primavera, al brotar, esa yemas se desarrolle una agalla en cuyo interior, se desarrolla el parásito. En el vivero, Dryocosmus está en estado de larva en Mayo-Junio, prepupa-adulto inmaduro en Junio, y en Julio emerge el adulto, siendo la emergencia en el exterior del vivero algo más tardía. En Julio-Agosto por tanto, se repite el proceso de puesta.

¿Qué ocurre cuando Torymus sinensis está presente en este escenario? Pues que las agallas no contienen sólo Dryocosmus, sino que sobre algunas de las larvas de Dryocosmus se está desarrollando la larva de Torymus. El adulto del parasitoide emergerá de las agallas secas de invierno en Abril-Mayo, cuando las nuevas agallas producidas por las larvas de Dryocosmus estén en pleno desarrollo. Torymus pondrá sus huevos en las agallas sobre las larvas de Dryocosmus impidiendo su emergencia en verano y limitando la infección del año siguiente. El sistema de producción del parasitoide en Italia, se basa por tanto, en la simple cosecha de las agallas parasitizadas en invierno, en zonas donde hay un elevado nivel de parasitización por parte del parasitoide. Las agallas se disponen en cajas de cría para esperar la emergencia de los adultos, seleccionarlos, sexarlos y expedirlos en tubos para su suelta.

En España el sector de la castañicultura está pendiente de la autorización por parte del MAPAMA para el uso de Torymus sinensis. Las autorizaciones que existen actualmente son únicamente para su uso experimental. En nuestro país por tanto, aún no es factible el sistema de producción italiano, por lo que se ha planteado un sistema de cría en vivero, en 2 macro jaulas aisladas con malla antitrips. En ellas se colocó en primavera de 2016 , la planta de castaño recriada en cantidad suficiente (5000 plantas). Posteriormente se introdujeron semanalmente desde principios de Junio hasta finales de Julio de 2016, agallas tomadas del campo, que se colocaron entre las plantas, provocando que en primavera de 2017 las plantas estén cuajadas de agallas, en mayor o menor medida según la distancia al foco de infección y el genotipo. Sobre estas agallas se han liberado en Mayo de 2017, 12 viales de Torymus sinensis que contiene cada uno 20 hembras y 12 machos. Al mismo tiempo, en la segunda jaula, se han introducido en la primavera de 2017 otras 5000 plantas, y se ha repitiendo el proceso de infección trayendo semanalmente agallas de Dryocosmus del exterior para disponer de planta con agalla sobre la que liberar los Torymus en la primavera del próximo año. Las agallas de las plantas parasitizadas esta primavera se cosecharán en invierno, y se colocarán en cajas de cría, para esperar la emergencia de los parasitoides que se destinarán a reinfección y a suministro de la Xunta de Galicia. 
Cuando visitamos en 2015 el Centro Técnico Interprofesional de las Frutas y Legumbres de Prigonrieux, en el sur de Francia donde tradicionalmente se ha trabajado con castaño, los franceses ya disponían de un ranking de susceptibilidad de los clones de uso más habitual tanto para fruto como para uso como portainjertos. Con el objetivo de disponer también de esa información de las variedades y clones españoles, aprovechando la infraestructura descrita anteriormente, hicimos un ensayo colocando 24 plantas de cada uno de los clones portainjertos y de las variedades de fruto, en 4 repeticiones de 6 individuos, distribuidas de manera aleatoria en torno a los diferentes focos de infección que se introdujeron en las jaulas. En la primavera de 2017 se han tomado datos de porcentaje de plantas infestadas y de $n .^{\circ}$ de agallas producidas en cada planta infestada, y cruzando ambos datos hemos construido un índice que nos ha permitido hacer un ranking de tolerancia a Dryocosmus. Como se aprecia en Figura 4, el clon de producción directa 90025 no presentó ninguna agalla en ninguna de las 24 plantas, por lo que podemos decir que es resistente. Le sigue el clon 90044, donde sólo 1 planta se infestó presentando 3 agallas. El portainjerto menos susceptible fue $\mathrm{C} 003$ donde se infectaron más del $80 \%$ de las plantas pero presentando un número muy bajo de agallas por planta, y las variedades menos sensible fueron Negral y Longal con un 88 y un $66 \%$ de las plantas infestadas respectivamente pero con muy pocas agallas por planta. En cualquier caso, hay que tener en cuenta que si bien cuando hablamos de híbridos hablamos de un solo genotipo, las variedades son policlonales en algunos casos, con lo que unos genotipos pueden ser sensibles y otros no.

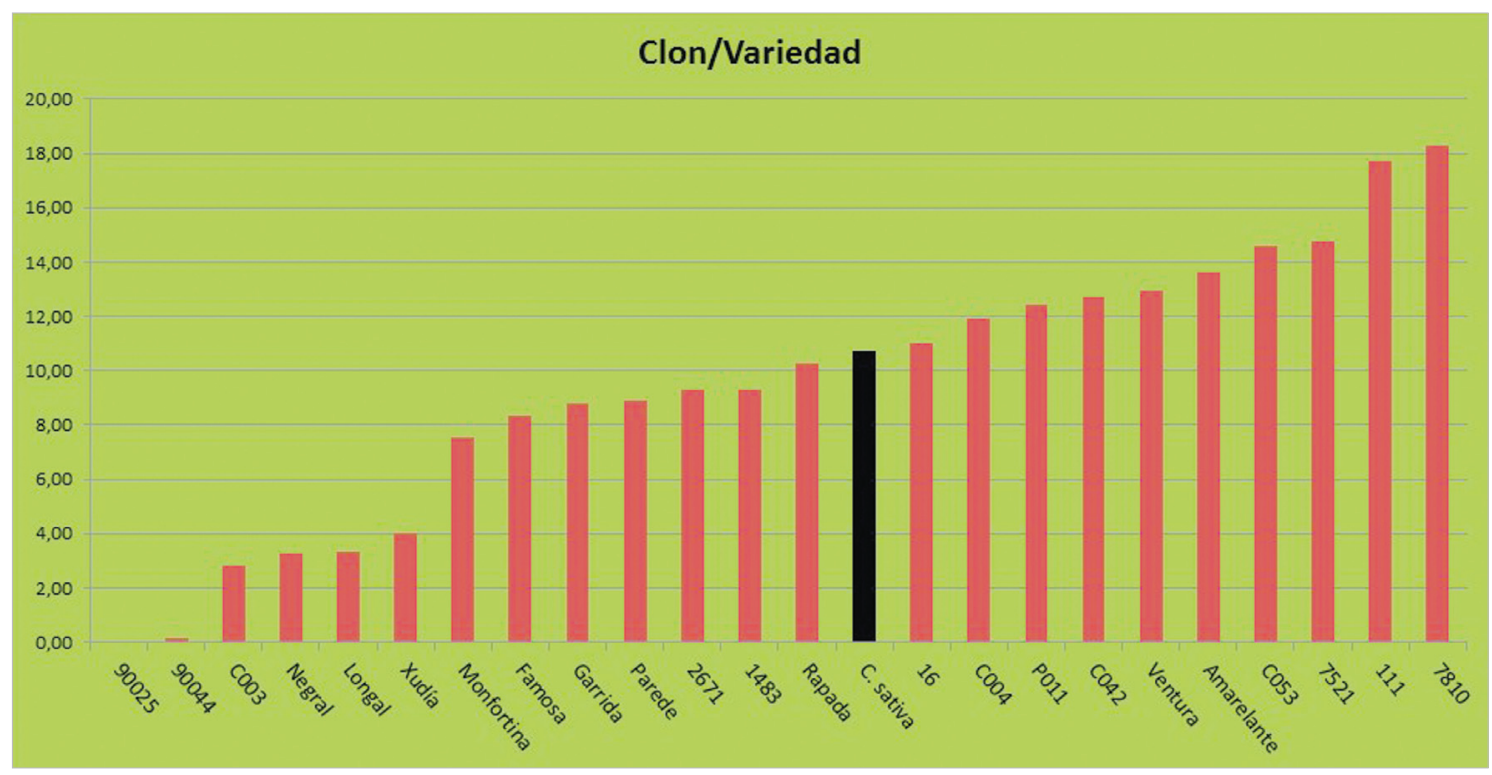

Figura 4. Ranking de clones y variedades de castaño por susceptibilidad al ataque de Dryocosmus kuriphilus.

Además se realizó la caracterización de los parasitoides autóctonos de nuestra zona, de modo que se recogieron agallas de invierno, en el mes de Marzo, y agallas de verano, en el mes de Mayo de 2016. Obviamente las agallas recogidas en invier- 
no correspondían a agallas aparecidas en los árboles en la primavera de 2015. La recogida se hizo en 4 árboles diferentes de 6 parcelas en torno al vivero. Las agallas se colocaron de manera individualizada por finca y árbol en cajas de cría y los insectos emergidos se enviaron a la Escuela de Forestales de Pontevedra para su identificación. Se identificaron 9 especies de parasitoides, todos ellos polífagos y por tanto no específicos de Dryocosmus. 4 de ellos aparecían tanto en las agallas de verano como en las de invierno, lo que parece sugerir que estos parasitoides tienen la estrategia de diferir su emergencia en un porcentaje de modo que unos emergen en verano y otros en la primavera siguiente. Estos pueden ser los que nos interesaran para parasitizar a Dryocosmus puesto que las emergencia de verano son demasiado tardías, de hecho coinciden con las de Dryocosmus, con lo que las puestas llegarían tarde para parasitizar al parásito en estado de larva. Sin embargo, y a pesar de tratarse de parasitoides bien implantados en el territorio, presentaron una tasa de parasitización muy baja, $0,52 \%$, sumando las tasas de las 4 especies, frente al más del 80\% que consigue Torymus en Italia en las zonas donde se ha implantado.

\section{Producción de Anaphes nitens para el control de Gonipterus platensis}

Esta producción se ha comenzado esta primavera, tras unos ensayos previos que pretendían optimizar para nuestras condiciones, el sistema de producción que inicialmente nos mostraron ENCE y SERPA (Principado de Asturias). Se ensayaron tres tipos de envases para la cría de Gonipterus y se seleccionaron las opciones de mayor producción y rendimiento, considerando el tiempo empleado en limpiar y renovar diariamente la comida de las cajas, y por tanto el número máximo de cajas que podían procesarse por semana. La opción seleccionada fue la caja de $30 \mathrm{~L}$ con la alimentación en agua y con una carga de 100 parejas. La producción diaria de ootecas, se le ofrece a Anaphes nitens para que hagan la puesta sobre ellas. Se realizaron ensayos con diferentes cargas de parasitoides/ooteca donde la variable medida fue el número de emergencias posteriores de Anaphes, y por tanto, el porcentaje de parasitización. La opción más productiva fue de 15 Anaphes (10 hembras y 5 machos) por cada 20 ootecas. El formato de liberación en campo es una bolsa de malla con 50 ootecas, y la ventana de liberación está entre los $4 \mathrm{~d}$ en se produce la muerte de los Anaphes y los 17-20 días desde la parasitización, en que empiezan a emerger la nueva generación de Anaphes. La opción de frigoconservar las ootecas a $8^{\circ} \mathrm{C}$ en ese lapso de tiempo hasta la liberación, también ha dado buenos resultados.

\section{Trabajos futuros en lucha biológica}

En 2017, en el contexto una nueva encomienda de la Xunta de Galicia, se comenzará a trabajar en el desarrollo del sistema de cría de otro parasitoide de G. platensis, Entedon magnificus, un himenóptero endoparásito de las larvas del gorgojo. Este pa- 
rasitoide hace la puesta sobre las larvas L4 del gorgojo, que sirven de alimento a las larvas del parasitoide. Por lo tanto, nuestro objetivo es conseguir cerrar el ciclo de cría del gorgojo en cautividad para disponer de larvas L4 sobre las que criar el Entedon.

Y por otro lado, en el contexto de la misma encomienda se ensayarán dos organismos para el control biológico de Altica quercetorum, un crisomélido cuyas larvas producen daños en el parénquima foliar de los robles del país (Quercus robur). Se ensayará el efecto producido por el hongo Beauveria bassiana y por el nemátodo entomopatógeno Heterorhabditis megidis en el control del insecto durante la hibernación y la pupación en el suelo.

\section{Puesta a punto de métodos de teledetección para el control sanitario de masas forestales}

TRAGSA está trabajando para desarrollar metodologías y protocolos de trabajo para detección temprana, caracterización y seguimiento del estado fitosanitario de las masas forestales, empleando datos de campo e imágenes multiespectrales, térmicas y visibles capturadas desde satélite y RPAS (Remote Piloted Aerial Systems). Comenzamos en estos trabajo colaborando con TRAGSATEC en el proyecto SUDOE FORRISK, donde junto con la U. de Córdoba y la empresa Zumain detectó Armillaria mellea en pinares empleando las imágenes del satélite WorldView 2 y RPAS equipados con sensores multiespectrales y RGB. En este trabajo se obtuvieron buenas correlaciones para los índices de vegetación GNDVI (green normalised difference vegetation index) y NDVI (normalised difference vegetation index), y los parámetros biofísicos de concentración en clorofila ( $\mathrm{r}$ entre 0,65 y 0,71$)$ y el índice de área foliar LAI ( $\mathrm{r}$ mayores de 0,8$)$. Y, también entre estos índices y la altura del árbol ( $\mathrm{r}$ entre 0,64 y 0,77 ) y diámetro del tronco ( $\mathrm{r}$ entre 0,69 y 0,72 ) que podrían estar mostrando el efecto acumulado de la afección.

Este trabajo ya ha concluido pero hay nuevos proyectos en marcha en TRAGSATEC, como el proyecto DATABIO (H2020), que se centrará en la detección y caracterización de la seca en la encina y de los daños del gorgojo en el eucalipto, y el proyecto PLURIFOR (Interreg SUDOE). En estos proyectos, el grupo TRAGSA colaborará con CETEMAS, ISA, INIAV, U. Valladolid, ENCE, RAIZ, y CICYTEX.

\section{Referencias bibliográficas}

Bouhier, A., 1979. La Galice. Essai geographique d'analyse et d'interpretation d'un viex complex agraire. Université de Poitiers, Imprimerie La Roche-sur-Yon. 1516 pp. Vendée, France.

Cuenca, B., Fernández, M.R., Ocaña, L., Salinero, C., Pintos, C., Mansilla, J.P., Rial, C., 2008. Selection of Castanea sativa Mill. for resistance to Phytophthora cinnamomi: testing of selected clones. In IV International Chestnut Symposium 844 (pp. 395-404).

Cuenca, B., Luquero, L., Ocaña, L., 2013. Nuevos Materiales Forestales de Reproducción de Castanea sp. de categoría cualificado. Actas del $6^{\circ}$ Congreso Forestal Español. Sociedad 
Española de Ciencias Forestales, Junta de Castilla y León, eds. Vitoria. Disponible en: http://congresoforestal.es

Chandelier, A., Husson, C., Druart, P., Marçais, B.C., 2015. Assessment of inoculation methods for screening black alder resistance to Phytophthora xalni. Plant Pathology, 65(3), 441-450.

Haque, M.M.U., Martín-García, J., Diez, J.J., 2015. Variation in pathogenicity among the three subspecies of Phytophthora alni on detached leaves, twigs and branches of Alnus glutinosa. Forest Pathology, 45(6), 484-491.

Jung, T., Blascgke, H., Newman, P., 1996. Isolation, identification and pathogenity of Phytophthora species from declining oak stands. Eur J Forest Pathol 26, 253.272.

Rial, C., Barros, A., Mansilla, J.P., Pintos, C., Cuenca, B., 2007. Variaciones en la susceptibilidad a Phytophthora cinnamomi de diferentes clones de castaño: comparación de dos métodos de inoculación. Reunión Científica del Grupo de Trabajo de Sanidad Forestal de la Sociedad Española de Ciencias Forestales, Palencia, Spain.

Robin, C., Morel, O., Vettraino, A.M., Perlerou, C., Diamandis, S., Vannini, A., 2006. Genetic variation in susceptibility to Phytophthora cambivora in European chestnut (Castanea sativa). Forest Ecology and Management, 226. 199-207.

Salesses, G., Chapa, J. and Chazerans, P., 1993. Amélioration génétique du châtaignier. Arb. Fruit, $58: 23-31$.

Solla, A., Aguín, O., Cubera, E., Sampedro, L., Mansilla, J.P., Zas, R., 2011. Survival time analysis of Pinus pinaster inoculated with Armillaria ostoyae: genetic variation and relevance of seed and root traits. Eur J Plant Pathol 130, 477-488 